\title{
New Discrete Tanaka Sugeno Kang Fuzzy Systems Characterization and Stability Domain
}

\author{
Mohamed Benrejeb, Dhaou Soudani, Anis Sakly, Pierre Borne
}

\begin{abstract}
In this paper, an analytical approach to characterize discrete Tanaka Sugeno Kang (TSK) fuzzy systems is presented. This characterization concerns the choice of the adequate conjunctive operator between input variables of discrete TSK fuzzy models, t-norm, and its impact on stability domain estimation. This new approach is based on stability conditions issued from vector norms corresponding to a vector-Lyapunov function. In particular, second order discrete TSK models are considered and this work concludes that Zadeh's t-norm, logic product min, gives the largest estimation of stability domain.
\end{abstract}

Keywords: Discrete nonlinear systems, discrete TSK fuzzy model, t-norm, stability domain, vector norm, arrow form matrix.

\section{Introduction}

Fuzzy control of systems presents a major interest in several applications including industrial ones. However, closed loop system properties are not easily understood and the design of the fuzzy system is generally based on intuitive approaches.

Different fuzzy control strategies exist. In particular, TSK fuzzy approach permits the description and the control of a system by defining different models related via a rule base.

The stability of TSK systems has been one of the central issues and is subject of many works either in the continuous case or in the discrete one. To do this, different approaches are considered mainly based on Lyapunov functions $[5,10,12]$. In particular, the Linear Matrix Inequality (LMI) formulation is used [11] and, according to the considered system, permits the stability problem resolution. In our previous work [1], the used approach is led through the convergence of a regular vector norm [3]. The vector norm approach, based on the comparison and overevaluating principle, has a major advantage that it deals with a very large class of systems, since no restrictive assumption is made on the matrices of state equations. So, in [1], the approach estimates the stability domain of continuous TSK fuzzy systems and its dependence on the choice of the conjunctive operator between inputs.

In this way, many authors have presented and analyzed several of these operators defined by t-norms and so said t-operators $[4,6,7]$. In particular, in [6], six t-norms are used to represent this connective operator in the inverse pendulum control. In another work [9], are used the same t-norms to study their impact on fuzzy control performances of a second order process by defining a performance criterion based on error measurement of the closed loop system. However, then exist other operators to represent this connective such as mean operators developed in [14].

Except [1], all these studies don't treat the influence of the choice of the conjunctive operator between inputs on the stability conditions obtained and often only prod or min operators are used without theoretical argumentation and just for simplification reasons.

Therefore, in this way the study will be considered and constitutes a generalization of [1] in the discrete case seeing the soft implementation nature of this control strategy.

By exploiting conditions or hypothesis obtained from stability analysis of discrete TSK fuzzy systems based on vector norms approach, the influence of the choice of t-norm is studied. Similarly to [1], only second order TSK fuzzy models are considered for simplification reason.

In the next section, is presented the structure of discrete TSK fuzzy models. In section 3, several definitions dealing with t-norm notion as well as its properties are given. In section 4, sufficient stability conditions of these TSK systems based on vector norms approach are presented. Then, the study of the 
impact of the choice of t-norm on the proposed stability conditions will be considered in section 5 . In particular, second order TSK fuzzy models are considered. A DC motor is studied in section 6 as an application example validating this study. Finally, some concluding remarks are pointed out.

\section{TSK discrete models description}

An $r$-order TSK discrete fuzzy model of an $n$-order nonlinear system to be controlled is given by a rule base where the $i^{\text {th }}$ rule is in the form:

$$
\begin{aligned}
& R_{i}: \text { IF } x_{1} \text { is } G_{1}^{i} A N D x_{2} \text { is } G_{2}^{i} A N D \cdots A N D x_{n} \text { is } G_{n}^{i} \\
& \quad \text { THEN }\left\{\begin{array}{l}
x(k+1)=A_{i} x(k)+B_{i} u(k) \\
y(k)=C_{i} x(k)
\end{array} \quad i=1,2, \ldots, r\right.
\end{aligned}
$$

where $G_{j}^{i}, j=1,2, \ldots, n$, is the $i^{t h}$ fuzzy set of the state vector $x_{j}$.

The state vector $x \in \mathfrak{R}^{n}$, the control input $u \in \mathfrak{R}$ and the matrices $A_{i}, B_{i}$ and $C_{i}$ are of appropriate dimensions.

According to the Parallel Distributed Compensation (PDC) concept [13], the rule base $R_{i}^{\prime}$ of the fuzzy controller stabilizing the former system is in the form:

$$
\begin{gathered}
R_{i}^{\prime}: I F x_{1} \text { is } G_{1}^{i} A N D x_{2} \text { is } G_{2}^{i} A N D \cdots A N D x_{n} \text { is } G_{n}^{i} \\
\operatorname{THEN} u(k)=-K_{i} x(k) \quad i=1,2, \ldots, r
\end{gathered}
$$

with $K_{i}=\left[k_{i}^{1}, k_{i}^{2}, \ldots, k_{i}^{n}\right]$.

By substituting $u$ in the equation (1) above, it comes:

$$
x(k+1)=\sum_{i=1}^{r} \sum_{j=1}^{r} h_{i} h_{j}\left(A_{i}-B_{i} K_{j}\right) x(k)
$$

with:

$$
\begin{gathered}
h_{i}=w_{i} / \sum_{i=1}^{r} w_{i} \\
w_{i}=T\left(G_{1}^{i}\left(x_{1}\right), G_{2}^{i}\left(x_{2}\right), \ldots, G_{n}^{i}\left(x_{n}\right)\right)
\end{gathered}
$$

and $T$ a t-norm.

When linear models of the system to be controlled are considered in the controllable form given by:

$$
A_{i}=\left[\begin{array}{cccc}
0 & 1 & \cdots & 0 \\
\vdots & \ddots & \ddots & \vdots \\
0 & \cdots & 0 & 1 \\
-a_{i}^{1} & \cdots & -a_{i}^{n-1} & -a_{i}^{n}
\end{array}\right] \text { and } B_{i}=B=\left[\begin{array}{c}
0 \\
\vdots \\
0 \\
1
\end{array}\right]
$$

relation (3) becomes:

$$
\begin{aligned}
& x(k+1)=\sum_{i=1}^{r} \sum_{j=1}^{r} h_{i} h_{j}\left(A_{i}-B_{i} K_{j}\right) x(k) \\
& =\sum_{i=1}^{r} \sum_{j=1}^{r} h_{i} h_{j}\left(A_{i}-B K_{j}\right) x(k) \\
& =\sum_{i=1}^{r} \sum_{j=1}^{r} h_{i} h_{j} A_{i} x(k)-\sum_{i=1}^{r} \sum_{j=1}^{r} h_{i} h_{j} B K_{j} x(k) \\
& =\sum_{i=1}^{r} h_{i} \sum_{j=1}^{r} h_{j} A_{i} x(k)-\sum_{j=1}^{r} h_{j} \sum_{i=1}^{r} h_{i} B K_{j} x(k) \\
& =\sum_{i=1}^{r} h_{i} A_{i} x(k)-\sum_{j=1}^{r} h_{j} B K_{j} x(k)
\end{aligned}
$$


then finally:

$$
x(k+1)=\sum_{i=1}^{r} h_{i}\left(A_{i}-B K_{i}\right) x(k)
$$

\section{T-norms}

An important task to be performed in the design of TSK fuzzy systems is the choice of the conjunctive operator materializing the connective $A N D$ between input variables in the rule base and so corresponding to the intersection operation between fuzzy subsets relatively to different inputs.

Often, this operator is defined by a t-norm $T$, whose definition and proprieties are presented as follows.

Definition 1. A triangular norm (t-norm) is a function $T:[0,1] \times[0,1] \rightarrow[0,1]$ verifying for every $u, v, w$ and $t$ of $[0,1]$ :

i) $T(u, v)=T(v, u)$ (commutativity)

ii) $T(u, T(v, w))=T(T(u, v), w)$ (associativity)

iii) $T(u, v) \leq T(w, t)$ si $u \leq w$ et $v \leq t$ (monotonicity)

iv) $T(u, 1)=u$ (one identity)

$\min$ operator is the largest of all possible t-norms, $T(u, v) \leq \min (u, v)$.

The intersection operation between fuzzy subsets is defined by a t-norm $T$ such as:

$$
C=A \cap B, \text { then } \forall x \in X \mu_{C}(x)=T\left(\mu_{A}(x), \mu_{B}(x)\right)
$$

The commonly encountered t-norms are given in table 1 .

Table 1: Main t-norms

\begin{tabular}{|l|l|}
\hline \multicolumn{1}{|c|}{ t-norm } & \multicolumn{1}{c|}{ Name } \\
\hline $\min (u, v)$ & Zadeh (logical operators) \\
\hline$u v$ & Bandler (probabilistic operators) \\
\hline $\max (u+v-1,0)$ & Lukasiewicz (bounded operators) \\
\hline$\left\{\begin{array}{l}u \text { if } v=1 \\
v \text { if } u=1 \\
0 \text { otherwise }\end{array}\right.$ & Weber (drastic operators) \\
\hline
\end{tabular}

Definition 2. A t-norm $T$ is said archimedean if and only if $T(u, v)$ is continuous and $T(u, u)<u$ for each $u$ of ]0,1[. Moreover, $T$ is said strict archimedean if and only if $T(u, v)<T(w, t)$ as soon as $u<w$ and $v<t$.

Any function $T:[0,1] \times[0,1] \rightarrow[0,1]$ is an archimedean t-norm if and only if it exists a continuous decreasing function $f:[0,1] \rightarrow[0,+\infty[$ such that $f(1)=0$ satisfying :

$$
T(u, v)=f^{-1 *}(f(u)+f(v))
$$

where $f^{-1 *}$ is the pseudo-inverse of $f$ such as $f^{-1 *}=\left(\begin{array}{l}f^{-1}(w) \text { if } w \in[0, f(0)[ \\ 0 \text { if } w \in[f(0),+\infty[\end{array}\right.$. 
Besides, $T$ is strict if and only if $f(0)=+\infty$.

$f$ is said the additive generator of the t-norm $T$.

The additive generators associated to main parameterized archimedean t-norms are presented in table $2[14]$.

Table 2: Main parametrized t-norms and their additive generators

\begin{tabular}{|c|c|c|c|}
\hline t-norm & Generator $f(u)$ & Parameter & Name \\
\hline$\frac{u v}{\gamma+(1-\gamma)(u+v-u v)}$ & $\begin{array}{l}\frac{1}{\gamma} \log \left[\frac{\gamma+(1-\gamma) u}{u}\right] \\
\text { Strict Archimedean }\end{array}$ & $\gamma>0$ & Hamacher \\
\hline$\frac{1}{\left[\left(\frac{1}{u}\right)^{p}+\left(\frac{1}{v}\right)^{p}-1\right]^{\frac{1}{p}}}$ & $\begin{array}{c}\frac{1}{(1+u)^{\frac{1}{p}}} \\
\text { Strict Archimedean }\end{array}$ & $p>0$ & Schweizer \& Sklar \\
\hline$\frac{1}{1+\left[\left(\frac{1}{u}-1\right)^{\lambda}+\left(\frac{1}{v}-1\right)^{\lambda}\right]^{\frac{1}{\lambda}}}$ & $\begin{array}{c}\frac{1}{1+u^{\frac{1}{\lambda}}} \\
\text { Strict Archimedean }\end{array}$ & $\lambda>0$ & Dombi \\
\hline $1-\min \left[1,\left((1-u)^{\omega}+(1-v)^{\omega}\right)^{\frac{1}{\omega}}\right]$ & $\begin{array}{l}\qquad(1-u)^{\omega} \\
\text { Archimedean no strict }\end{array}$ & $\omega>0$ & Yager \\
\hline$\frac{u v}{\max (u, v, \alpha)}$ & No archimedean & $\alpha \in[0,1]$ & Dubois \& Prade \\
\hline
\end{tabular}

\section{New stability conditions}

In [2], a change of base of (6) under the arrow form give:

$$
x(k+1)=\sum_{i=1}^{r} h_{i} M_{i} x(k)
$$

where $M_{i}$ is a matrix in the arrow form and $P$ is the corresponding passage matrix:

$$
M_{i}=P^{-1}\left(A_{i}-B K_{i}\right) P
$$




$$
M_{i}=\left[\begin{array}{ccccc}
\alpha_{1} & 0 & \cdots & 0 & \beta_{1} \\
0 & \ddots & \ddots & \vdots & \vdots \\
\vdots & \ddots & \ddots & 0 & \vdots \\
0 & \cdots & 0 & \alpha_{n-1} & \beta_{n-1} \\
\gamma_{i}^{1} & \cdots & \cdots & \gamma_{i}^{n-1} & \gamma_{i}^{n}
\end{array}\right] \text { and } P=\left[\begin{array}{ccccc}
1 & 1 & \cdots & 1 & 0 \\
\alpha_{1} & \alpha_{2} & \cdots & \alpha_{n-1} & 0 \\
\alpha_{1}^{2} & \alpha_{2}^{2} & \cdots & \alpha_{n-1}^{2} & \vdots \\
\vdots & \vdots & \cdots & \vdots & 0 \\
\alpha_{1}^{n-1} & \alpha_{2}^{n-1} & \cdots & \alpha_{n-1}^{n-1} & 1
\end{array}\right]
$$

with:

$$
\begin{aligned}
& \beta_{j}=\prod_{\substack{q=1 \\
q \neq j}}^{n-1}\left(\alpha_{j}-\alpha_{q}\right)^{-1} \forall j=1,2, \ldots, n-1 \\
& \left\{\begin{array}{l}
\gamma_{i}^{j}=-P_{i}\left(\alpha_{j}\right) \forall j=1,2, \ldots, n-1 \\
P_{i}(\lambda)=\lambda^{n}+\sum_{q=0}^{n-1}\left(a_{i}^{q+1}+k_{i}^{q+1}\right) \lambda^{q}
\end{array}\right. \\
& \gamma_{i}^{n}=-\left(a_{i}^{n}+k_{i}^{n}\right)-\sum_{j=1}^{n-1} \alpha_{j}
\end{aligned}
$$

The application of the classical Borne-Gentina criterion [3] leads to the following theorem.

Theorem 3. The discrete system described by (7) is asymptotically stable if there exist $0<\alpha_{j}<1, \alpha_{j} \neq \alpha_{k}, \forall j \neq k$, such as $\forall x \in S$ :

$$
\begin{aligned}
& \text { i) } 1-\left|\alpha_{j}\right|>0 \forall j=1,2, \ldots, n-1 \\
& \text { ii) } \quad 1-\left|\sum_{i=1}^{r} h_{i} \gamma_{i}^{n}\right|-\sum_{j=1}^{n-1}\left|\sum_{i=1}^{r} h_{i} \gamma_{i}^{j} \beta_{j}\right|\left(1-\left|\alpha_{j}\right|\right)^{-1}>0
\end{aligned}
$$

If $S=\Re^{n}$, the stability is global.

Furthermore, if there exist $\alpha_{j}, j=1,2, \ldots, n-1$, such as :

$$
\begin{aligned}
\text { i) } & 0<\alpha_{j}<1 j=1,2, \ldots, n-1 \\
\text { ii) } & \sum_{i=1}^{r} h_{i} \gamma_{i}^{n}>0 \\
\text { iii) } & \sum_{i=1}^{r} h_{i} \gamma_{i}^{j} \beta_{j}>0 j=1,2, \ldots, n-1
\end{aligned}
$$

then the previous theorem can be simplified to the following corollary.

Corollary 4. The discrete system described by (7) is asymptotically stable if there exist $0<\alpha_{j}<1$, $\alpha_{j} \neq \alpha_{k}, \forall j \neq k$, such as $\forall x \in S$ :

$$
B P H<0
$$

where matrices $B \in \mathfrak{R}^{n \times n}, P \in \mathfrak{R}^{n \times r}$ and $H \in \mathfrak{R}^{r \times 1}$ are such as :

$$
B=\left[\begin{array}{cccc}
\beta_{1} & 0 & \cdots & 0 \\
0 & \ddots & \ddots & \vdots \\
\vdots & \cdots & \beta_{n-1} & 0 \\
0 & \cdots & 0 & -1
\end{array}\right], P=\left[\begin{array}{ccc}
P_{1}\left(\alpha_{1}\right) & \cdots & P_{r}\left(\alpha_{1}\right) \\
\vdots & \cdots & \vdots \\
P_{1}\left(\alpha_{n-1}\right) & \cdots & P_{r}\left(\alpha_{n-1}\right) \\
P_{1}(1) & \cdots & P_{r}(1)
\end{array}\right] \text { and } H=\left[\begin{array}{c}
h_{1} \\
\vdots \\
h_{r}
\end{array}\right]
$$

If $S=\mathfrak{R}^{n}$, the stability is global.

According to the choice of the coefficients $\alpha_{j}$, different conditions on the parameters $h_{i}$ can be obtained. 


\section{T-norm influence on the proposed stability conditions}

Let us consider the following classical fuzzy partition of a second order TSK model given by figure 1, with:

$$
\mu_{i}=G_{i}^{1}\left(x_{i}\right) \quad i=1,2, \ldots, n
$$

and:

$$
G_{i}^{1}\left(x_{i}\right)=\min \left(1, \max \left(0, \frac{L_{i}-x_{i}}{2 L_{i}}\right)\right) \text { and } G_{i}^{2}\left(x_{i}\right)=1-G_{i}^{1}\left(x_{i}\right)
$$

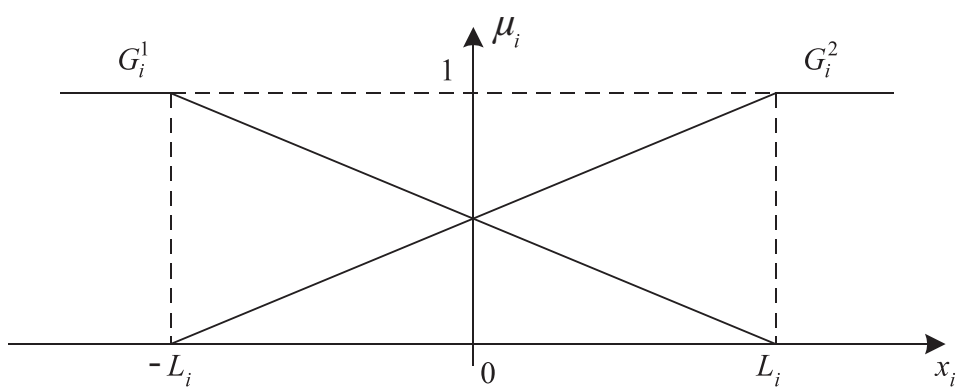

Figure 1: Fuzzy partition of state variables for $r=2$

Then, the whole fuzzy state space considered is the open subset $\Omega=]-L_{1}, L_{1}[\times]-L_{2}, L_{2}[\times \ldots \times]-L_{n}, L_{n}[$. So, the notion of global stability, whenever used in the literature on fuzzy control, refers to local stability within a such domain [8].

For second order TSK model, i.e. $r=2$, then $h_{2}=1-h_{1}$ and:

$$
h_{1}=\frac{w_{1}}{w_{1}+w_{2}}=\frac{T\left(\mu_{1}, \mu_{2}, \ldots, \mu_{n}\right)}{T\left(\mu_{1}, \mu_{2}, \ldots, \mu_{n}\right)+T\left(1-\mu_{1}, 1-\mu_{2}, \ldots, 1-\mu_{n}\right)}
$$

where $T$ is a t-norm. Propositions [1]

1. Let $\mu_{1}, \mu_{2}, \ldots, \mu_{n}$ and $\mu_{\max } \in[0,1]$ such as $\mu_{1} \leq \mu_{\max }, \mu_{2} \leq \mu_{\max }, \ldots$, and $\mu_{n} \leq \mu_{\max }$.

Then, we obtain:

$$
h_{1}\left(\mu_{1}, \mu_{2}, \ldots, \mu_{n}\right) \leq h_{1}\left(\mu_{\max }, \mu_{\max }, \ldots, \mu_{\max }\right)
$$

This proposition allows the determination of the domain $S$ by its characteristic point $M$ in figure 2 .

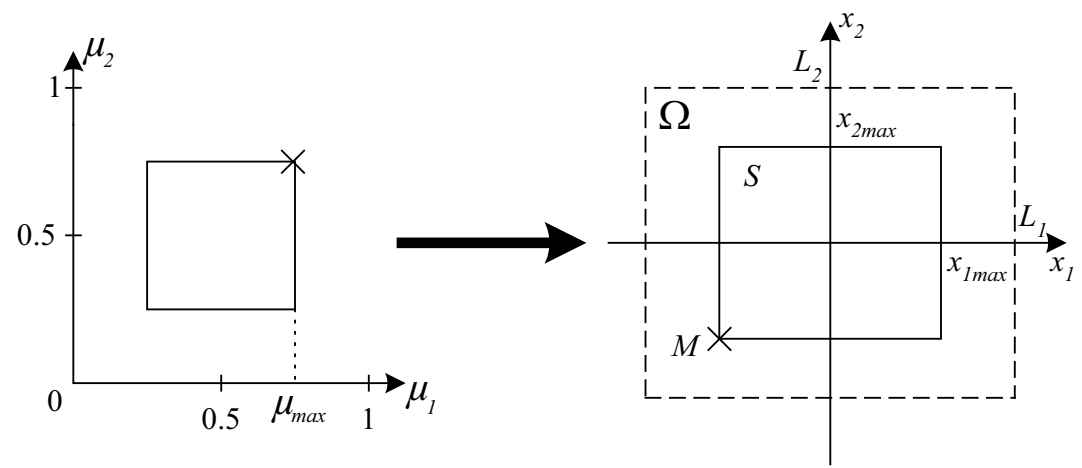

Figure 2: Correspondence between the state space variables domain and the membership values domain 
2. Let us note $\varphi_{n}(\mu)=T\left(\mu_{1}, \mu_{2}, \ldots, \mu_{n}\right)$ when $\mu_{1}=\mu_{2}=\ldots=\mu_{n}=\mu$. $\varphi_{n}$ is an increasing function on $[0,1]$ with $\varphi_{n}(0)=0$ and $\varphi_{n}(1)=1$.

If the following conditions are satisfied :

i) $\varphi_{n}$ is continuous

ii) $\forall \mu>0, \varphi_{n}(\mu) \neq 0$

iii) $\left.\left.\forall \mu_{1}, \mu_{2} \in\right] 0,1\right]$ with $\mu_{2}>\mu_{1}, \frac{\varphi_{n}\left(\mu_{2}\right)}{\mu_{2}} \geq \frac{\varphi_{n}\left(\mu_{1}\right)}{\mu_{1}}$

then we have:

$$
\left\{\begin{array}{l}
h_{1}(\mu, \mu, \ldots, \mu) \geq \mu \text { if } \mu \geq 0.5 \\
h_{1}(\mu, \mu, \ldots, \mu) \leq \mu \text { if } \mu \leq 0.5
\end{array}\right.
$$

This proposition means that for a given $\mu$, the smallest coefficient $h_{1}$ is obtained with the t-norm of Zadeh, the logic product $\mathrm{min}$; and so for the condition (22) we obtain the largest rectangular form domain $S$ with this t-norm by taking $\mu_{\max }=c$.

3. Conditions (23) of the proposition 2 are satisfied for each strict archimedean t-norm such as $\varphi_{n}(\mu)$ is derivable on $] 0,1]$.

The proposed approach is illustrated by considering the following parameterized t-norms:

- the Hamacher one: $T\left(\mu_{1}, \mu_{2}\right)=\frac{\mu_{1} \mu_{2}}{\gamma+(1-\gamma)\left(\mu_{1}+\mu_{2}-\mu_{1} \mu_{2}\right)}$ for $\gamma>0$,

- the Yager one: $T\left(\mu_{1}, \mu_{2}\right)=1-\min \left(1,\left(\left(1-\mu_{1}\right)^{\omega}+\left(1-\mu_{2}\right)^{\omega}\right)^{\frac{1}{\omega}}\right)$ for $\omega>0$,

- and the Dubois one: $T\left(\mu_{1}, \mu_{2}\right)=\frac{\mu_{1} \mu_{2}}{\max \left(\mu_{1}, \mu_{2}, \alpha\right)}$ for $\alpha \in[0,1]$.

\section{Application example}

As an example, let consider in figure 3 the case of a DC motor with two linear models $G_{1}(p)$ and $G_{2}(p)$ with $\delta_{1}=0.5$ and $\delta_{2}=1$.

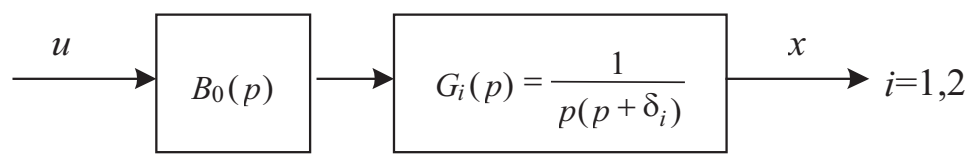

Figure 3: DC motor discrete models

$B_{0}(p)=\frac{1-e^{-T_{e} p}}{p}$ is a zero order holder and $T_{e}=0.2 \mathrm{~s}$ is the sampling time.

The discrete models of the DC motor are given by the following z-transmittances:

$$
G_{i}(z)=\frac{N_{i}(z)}{(z-1)\left(z-\psi_{i}\right)} \quad i=1,2
$$

with $\psi_{i}=e^{-\delta_{i} T_{e}}$ and $N_{i}(z)=\frac{\delta_{i} T_{e}+e^{-\delta_{i} T_{e}}-1}{\delta_{i}^{2}} z+\frac{1-e^{-\delta_{i} T_{e}}\left(1+\delta_{i} T_{e}\right)}{\delta_{i}^{2}}$.

The two discrete DC linear models are considered in the following controllable form:

$$
A_{1}=\left[\begin{array}{cc}
0 & 1 \\
-0.905 & 1.905
\end{array}\right] \quad, \quad A_{2}=\left[\begin{array}{cc}
0 & 1 \\
-0.819 & 1.819
\end{array}\right] \quad \text { and } \quad B=\left[\begin{array}{l}
0 \\
1
\end{array}\right]
$$

We suppose that for particular constraints the choice of $K_{i}$ is imposed such that the pole placement is different for the two models by taking: 


$$
K_{1}=\left[\begin{array}{ll}
-0.7 & 1.2
\end{array}\right] \text { and } K_{2}=\left[\begin{array}{ll}
-1.1 & 1.2
\end{array}\right]
$$

According to [3], the minimal overvaluing matrix relatively to the regular vector norm $p$ :

$$
p(x)=\left[\left|x_{1}\right|,\left|x_{2}\right|\right]^{T}
$$

is such as:

$$
M(.)=\left[\begin{array}{cc}
|\alpha| & 1 \\
\left|\gamma_{1}\right| & \left|\gamma_{2}\right|
\end{array}\right]
$$

with

$$
\begin{aligned}
& \gamma_{1}=(0.486-0.086 \alpha) h_{1}+\alpha^{2}-0.619 \alpha-0.281 \\
& \gamma_{2}=0.086 h_{1}+0.619-\alpha
\end{aligned}
$$

Then, stability conditions deduced from the corollary are:

$$
\begin{array}{ll}
\text { i) } & 0<\alpha<1 \\
\text { ii) } & 0.086 h_{1}+0.619-\alpha>0 \\
\text { iii) } & (0.486-0.086 \alpha) h_{1}+\alpha^{2}-0.619 \alpha-0.281<0 \\
\text { iv) } & 0.4 h_{1}+0.1>0
\end{array}
$$

When i) is satisfied, relations ii) and iv) are too. Then condition iii) leads to the following inequality

$$
h_{1}<-\frac{\alpha^{2}-0.619 \alpha-0.281}{0.486-0.086 \alpha}=c
$$

When $0.5<c<1$, let $S$ a neighborhood of the equilibrium point 0 , included in $\Omega$, $\Omega=]-1,1[\times]-1,1[$, that verifies a such condition. $S$ is an overvaluing domain of the fuzzy system and an estimation of a symmetrical domain $S$ with respect to 0 imposes:

$$
1-c<h_{1}<c
$$

Now, consider the study of the impact of the t-norm $T$ on the width of the neighborhood $S$ of the equilibrium point 0 , and then the determination of the largest stability domain $D$ included in $S$ verifying the previous condition.

For $\alpha=0.345$, we obtain the maximal value of $\mathrm{c}, c=0.82$. Thus, the overvaluing matrix $M(\cdot)$ is constant:

$$
M(\cdot)=M=\left[\begin{array}{ll}
\alpha & 1 \\
0 & \alpha
\end{array}\right]
$$

whitch is triangular and not irreductible. However, for $c=0.80$, this irreductibility is skirted and for $\alpha=0.345$, its comes the following overvaluing matrix

$$
M=\left[\begin{array}{cc}
0.345 & 1 \\
0.01 & 0.343
\end{array}\right]
$$

whitch is irreductible, whose principal eigenvalue is $\lambda_{m}=0.446$, the corresponding vector is:

$$
u_{m}=\left[\begin{array}{lll}
9.88 & 1
\end{array}\right]^{T}
$$

and the largest estimated stability domain $D$ is such as

$$
D=\left\{x \in S / p^{T}(x) u_{m}=9.88\left|x_{1}\right|+\left|x_{2}\right| \leq x_{\max }\right\}
$$

where $S$ is the square form domain and $x_{\max }$ is its width. $S$ depends on the condition $h_{1}<c$ with $c=0.8$ and so on the t-norm $T$. 
Table 3: $\mu_{\max }$ and $x_{\max }$ corresponding to different t-norms

\begin{tabular}{|l|l|l|}
\hline t-norm & $\mu_{\max }$ & $x_{\max }$ \\
\hline Zadeh & 0.80 & 0.60 \\
\hline Bandler & 0.67 & 0.34 \\
\hline Hamacher $(\gamma=0)$ & 0.75 & 0.50 \\
\hline Hamacher $(\gamma=\infty)$ & 0.58 & 0.16 \\
\hline Yager $(\omega=2)$ & 0.62 & 0.24 \\
\hline Dubois $(\alpha=0.5)$ & 0.70 & 0.40 \\
\hline
\end{tabular}

For $n=2$, table 3 gives $\mu_{\max }$, corresponding to the membership values domain, and $x_{\max }$, corresponding to the square form domain $S$, with respect to different t-norms.

The results obtained in table 3 shows that for $n=2$, the greatest value of $\mu_{\max }$ and so the largest domain $S$ is obtained specifically for the logic product $\mathrm{min}$.

Figure 4 presents an estimation of the largest stability domain $D$ respectively for Zadeh and Bandler t-norms.

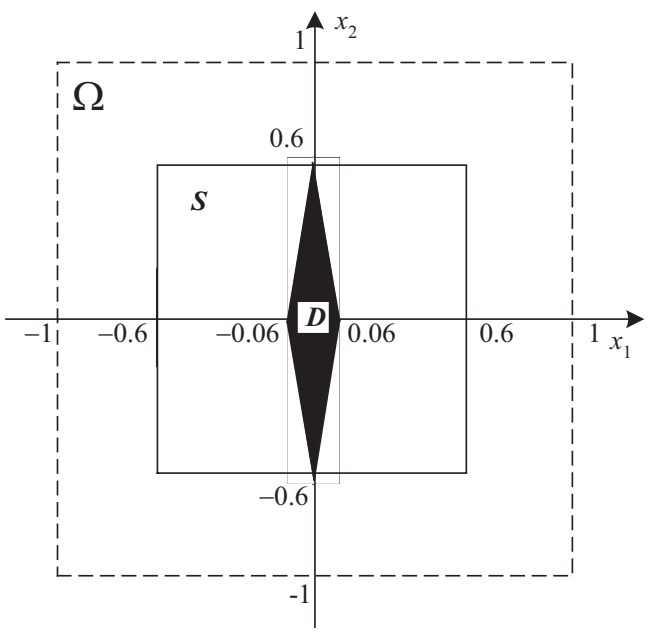

a) Zadeh's t-norm ( $\min )$

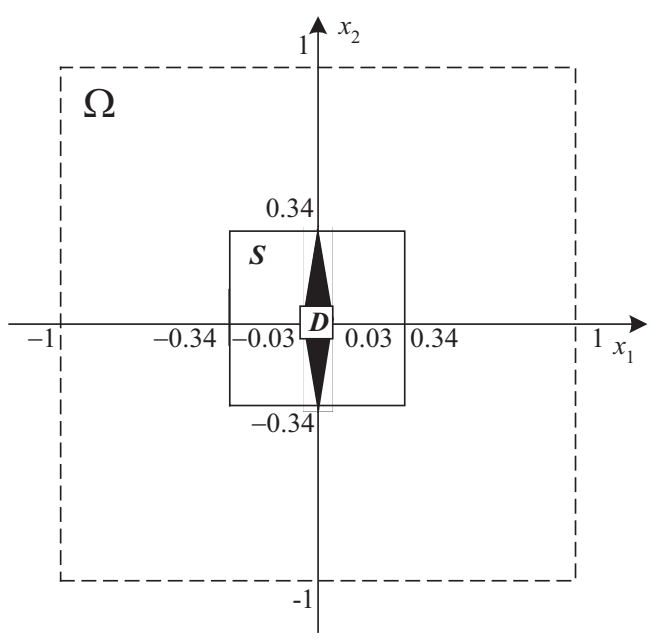

b) Bandler's t-norm (prod)

Figure 4: Stability domains obtained for two different t-norms

\section{Conclusion}

In this paper, we are interested in the stability study of discrete TSK fuzzy systems and the correspondent domain estimation relatively to the used conjunctive operator, materialized by a t-norm. The stability approach is based on vector norms. When second order TSK models are considered, a mathematical study permits to conclude that Zadeh's t-norm, $\min$ operator, yields to the largest stability domain. A DC motor with two discrete models is considered as an illustrative example and the estimated stability domains for different $\mathrm{t}$-norms confirm the former result.

However, it suits to remark that this study can be generalized for $r$-order TSK models, in the two 
cases continuous and discrete, with $r$ greater than 2 and then we must distinguish between the two cases, $r$ odd or even. For two cases, the stability study can be leaded in different symmetrical rectangular zones surrounding the equilibrium point, from the smallest to the largest.

\section{References}

[1] Benrejeb M., Sakly A., Ben Othman K. and Borne P.: Choice of Conjunctive Operator of TSK Fuzzy Systems and Stability Domain Study. To appear in MATCOM (MAThematics and COMputers and simulation Journal).

[2] Benrejeb M., Borne P. and Laurent F. : Sur une application de la représentation en flèche à l'analyse des processus. RAIRO Automatique, 16 (2). (1982) 133-146.

[3] Borne P., Richard J. P. and Radhy N. E. : Stabilité, stabilisation, régulation : approche par les normes vectorielles, Systèmes non linéaires, tome 2 : stabilité-stabilisation. coordonné par Fossard A. J. et Normand-Cyrot D., Responsable du tome : Borne P., 45-90. Editions Masson, Paris (1993).

[4] Butkiewicz B.S. : Control Error of Fuzzy System with Different Reasoning and Defuzzification Methods. 7th International Fuzzy Systems Association World Congress (IFSA), III. Praga, Czech (1997).

[5] Cao S. G., Rees N. W. and Feng G. : Quadratic stability analysis and design of continuous-time fuzzy control systems. Int. J. Syst. Sci., 27(2). (1996) 193-203.

[6] Cardenas E., Castillo J.C., Cordon O., Herrera F. and Peregrin A. : Applicability of T-norms in Fuzzy Control. Busefal, 61. (1995) 28-37.

[7] Gupta M.M. and Qi J. : Design of Fuzzy Logic Controllers Based on Generalized T-operators. Fuzzy Sets and Systems, 40. (1991) 473-489.

[8] Palm R., Driankov D. and Hellendoorn H. : Model Based Fuzzy Control. Springer-Verlag, Berlin Heidelberg (1997).

[9] Sakly A., Ben Othman K. and Benrejeb M. : Sur le Choix de l'Opérateur de Conjonction en Commande Floue Basé sur la Robustesse de Critères de Performances. $2^{\mathrm{ème}}$ Conférence Internationale JTEA, Tome 1, Sousse Nord (2002).

[10] Sugeno M. : On stability of fuzzy systems expressed by fuzzy rules with singleton consequences. IEEE Transactions on Fuzzy Systems, 7(2). (1999) 201-223.

[11] Tanaka K., Ikeda T. and Wang H.O. : Fuzzy Regulators and Fuzzy Observers : Relaxed Stability Conditions and LMI-based Designs. IEEE Transactions on Fuzzy Systems, 6(2). (1998) 1-16.

[12] Tanaka K. and Sugeno M. : Stability analysis and design of fuzzy control systems. Fuzzy sets and systems, 45. (1992) 135-156.

[13] Tanaka K. and Sano M. : A Robust Stabilization Problem of Fuzzy Control Systems and its Application to Backing up Control of a Truck-Trailer. IEEE Transactions on Fuzzy Systems, II. (1994) 119-134.

[14] Yager R. R. and Filev D. P. : Essentials of Fuzzy Modeling and Control. John Wiley \& Sons, Inc., USA (1994). 
Mohamed BENREJEB ${ }^{1}$, Dhaou SOUDANI ${ }^{1}$, Anis SAKLY $^{1}$, Pierre BORNE $^{2}$

${ }^{1}$ Ecole Nationale d'Ingénieurs de Tunis Unité de Recherche LARA-Automatique BP 37, Le Belvédère, 1002 Tunis, Tunisie

${ }^{2}$ Ecole Centrale de Lille, Cité scientifique Laboratoire d'Automatique, Génie Informatique et Signal BP 48, 59651 Villeneuve d'Ascq Cedex, France

E-mail: mohamed.benrejeb@enit.rnu.tn,dhaou.soudani@enit.rnu.tn, sakly_anis@yahoo.fr, pierre.borne@ec-lille.fr

Received: November 10, 2006 